\title{
Éditorial
}

\section{Conseil National Professionnel des chirurgiens-dentistes}

2015-2017: le Conseil National Professionnel des chirurgiens-dentistes (CNP-CD) existe depuis deux années au cours desquelles il a œuvré pour promouvoir la formation continue de nos confrères.

Il est né du constat fait par une grande majorité de la profession de l'insuffisance de la formation continue qui ne serait assurée que par le seul Développement Professionnel Continu (DPC).

C'est ainsi qu'en janvier 2015, un manifeste des associations scientifiques, des hospitalo-universitaires, des syndicats et bien sûr du Conseil de l'Ordre, dénommé « Manifeste pour la défense de la formation continue de notre profession » a précédé la création du CNP-CD en mai 2105, sous l'égide du Conseil national de l'Ordre.

\section{RÔLE ET MISSIONS DES CNP}

Selon les textes en vigueur, les CNP ont notamment pour mission :

- d'apporter leur concours aux instances de l'Agence nationale du développement professionnel continu (ANDPC), notamment pour la définition des critères d'évaluation des actions de développement professionnel continu proposées par les organismes ou les structures et l'élaboration des plans de contrôle annuel des actions de développement professionnel continu;

- d'assurer une veille sur les initiatives de terrain et les besoins des professionnels et de communiquer au ministre chargé de la Santé et au Haut conseil du développement professionnel continu (HCDPC) des professions de santé toutes informations ou propositions qu'ils jugent utiles pour évaluer l'intérêt et la pertinence des actions proposées et promouvoir le caractère collectif du développement professionnel continu, en secteur ambulatoire et en établissement de santé. 
L'avis des conseils nationaux professionnels peut également être sollicité :

- par le ministre chargé de la Santé et le HCDPC, sur les modifications éventuelles du DPC et l'évaluation de son impact sur les pratiques professionnelles;

- par les instances ordinales, les agences régionales de santé et les employeurs auprès desquels les professionnels justifient de leur engagement dans le DPC (essentiellement le service de santé des armées et les établissements de santé pour ce qui est de notre profession).

\section{ACTIONS DU CNP-CD}

La première action réalisée par le CNP-CD a été de proposer, sur la base des méthodes élaborées par la Haute autorité de santé, des orientations prioritaires qui lui paraissaient les plus adaptées pour la mise en œuvre du DPC des chirurgiens-dentistes.

Des orientations générales, pour l'ensemble de la profession, ainsi que des orientations spécifiques pour chacune des spécialités que compte la profession, ont été publiées dans un arrêté du 8 décembre 2015. Cependant, si certaines orientations générales ont bien été proposées par le CNP-CD, cela n'est pas le cas pour celles concernant les trois spécialités.

Ainsi pour l'ODF était formalisé le texte suivant :

«Chirurgien-dentiste spécialisé en orthopédie dento-faciale:

Orientation $n^{\circ} 1$ : innovation en orthopédie dento-faciale.

Orientation $n^{\circ} 2$ : nouvelles recommandations en orthopédie dento-faciale.

Orientation $n^{\circ} 3$ : gestion des risques associés aux actes et aux modalités de prise en charge en orthopédie dentofaciale. "

Le CNP-CD a donc proposé, il y a trois mois, les modifications suivantes :

"Chirurgien-dentiste spécialisé en orthopédie dento-faciale:

Orientation $n^{\circ} 1$ : mise en place des canines incluses.

Orientation $n^{\circ} 2$ : orthodontie: une pertinence médicale.

Orientation $n^{\circ} 3$ : modalités thérapeutiques innovantes en ODF. »

À noter que la majeure partie des orientations concernant les spécialistes en MBD et les omnipraticiens ont également fait l'objet de propositions de modifications par le CNP-CD.

Seules les orientations de DPC pour la chirurgie orale n'en ont pas eues puisqu'elles concernent aussi bien les chirurgiens-dentistes que les médecins. 
Nous restons donc dans l'attente de la parution de l'arrêté qui validera et officialisera ces modifications.

Le CNP-CD a également procédé à la nomination de huit membres titulaires et huit membres suppléants de la Commission scientifique indépendante des chirurgiens-dentistes, elle-même chargée d'évaluer les actions de DPC présentées par les organismes de DPC.

Le CNP-CD a élargi son bureau en nommant trois vice-présidents représentant chacun l'une des trois spécialités de notre profession (Alain Béry est vice-président représentant l’ODF).

\section{ÉVOLUTION DES CNP}

Un projet de décret a été soumis à l'analyse du CNO et du CNP, apportant des modifications, et pas des moindres, à la composition des CNP.

Le CNOCD et le CNP-CD ont eu la désagréable surprise de constater entre autres, à la lecture de ce projet de décret, que les instances ordinales et l'Université ne faisaient plus partie des membres du CNP, avec voix délibérative. Seul un « strapontin » leur était réservé, leur permettant de participer aux discussions avec seulement une voix consultative.

Le CNOCD et le CNP-CD ont fait part au ministère de leur incompréhension sur une telle composition, au regard des missions confiées aux CNP et sachant le rôle tant de l'Ordre que de l'Université dans la formation continue et le maintien des compétences des confrères.

Ce projet de décret a été rejeté par l'ensemble des CNP. Le ministère semble avoir compris nos réticences, et nous attendons donc la nouvelle écriture du texte après concertations.

Reste également à déterminer les modalités de financement des CNP, sachant que l'IGAS a rendu un rapport au ministère avec quelques pistes de financement.

\section{QUEL AVENIR POUR LES CNP?}

Dès sa création, le CNP-CD a estimé que le DPC sur trois ans ne pouvait à lui seul assurer une réelle et pertinente formation de nos confrères. Aussi souhaite-t-il ajouter au DPC une évaluation plus complète de la formation continue du chirurgien-dentiste en proposant une reconnaissance d'actions de formation hors DPC, assurées par des sociétés locales ou régionales. En effet, si le DPC est une obligation légale depuis la loi HPST du 21 juillet 2009, il convient de garder en mémoire que la formation continue reste une obligation déontologique, prévue à l'article R4127-214 du code de la santé publique.

C'est également la raison pour laquelle le CNP-CD a repris les orientations nationales, en tenant compte du fait que celles qui se rapportent aux spécialités ne sont accessibles qu’aux seuls spécialistes. 
Or nombreux sont les omnipraticiens, exerçant avec une orientation très marquée dans une discipline, à souhaiter suivre ce genre de formation. Ce n'est que dans un cadre hors DPC qu'ils pourront le faire.

Pour favoriser le processus de reconnaissance de telles formations hors DPC, le CNP a besoin de prérogatives élargies.

Nous avons présenté très récemment nos demandes de modification du décret ainsi que celle d'un élargissement de nos prérogatives lors d'une réunion au Ministère et espérons qu'elles seront prises en compte.

Paul Samakh

Président du CNP-CD

Les opinions émises n'engagent que leurs auteurs. 